Z. Epileptol. $2021 \cdot 34: 311-317$ https://doi.org/10.1007/s10309-021-00425-0 Angenommen: 9. Juni 2021

Online publiziert: 9. Juli 2021

(c) Der/die Autor(en) 2021

Sigrid Mues · Wenke Grönheit · Tim Wehner · Jörg Wellmer

Ruhr-Epileptologie, Klinik für Neurologie, Universitätsklinikum Knappschaftskrankenhaus Bochum, RuhrUniversität Bochum, Bochum, Deutschland

\title{
Tele-Epileptologie Ruhr: Zwischenevaluation eines telemedizinischen Modellprojektes
}

\section{Hintergrund und Fragestellung}

Neurologische Kliniken sind häufig mit Epilepsiepatienten bzw. Patienten mit anfallsartig auftretenden Symptomen anderer Ursache konfrontiert. Hinsichtlich der (Differenzial-)Diagnose und/ oder Therapie ergeben sich immer wieder Herausforderungen, die spezieller epileptologischer Expertise bedürfen. Epileptologen gehören jedoch nicht in jeder neurologischen Klinik zum Mitarbeiterstamm. Sich an telemedizinischen Klinik-zu-Klinik-Anwendungen im Bereich der Schlaganfallmedizin orientierend, hat die Ruhr-Epileptologie seit 2016 zunächst mit einer begrenzten Anzahl an Partnerkliniken eine digitale telemedizinische Infrastruktur mit allgemeinneurologischen Akutkliniken aufgebaut. Die Ruhr-Epileptologie ist ein universitäres Epilepsiezentrum für die Metropolregion Ruhr. Die Region stellt mit 5,1 Mio. Einwohnern und ca. 40 neurologischen Kliniken einen relevanten Markt für interhospitale, spezialistengeführte Konsilplattformen dar. Weiteres Ziel war die Schaffung eines Kommunikationsinstruments mit ausländischen Kliniken zur epilepsiechirurgischen Falldiskussion.

Zwei Jahre nach Start des Konsilbetriebs im September 2018 führten wir im Oktober 2020 eine Zwischenevaluation des Projektes durch. Die Ergebnisse sollen für das konkrete Projekt und darüber hinaus für vergleichbare Plattformen Hinweise geben, wo technische und organisatorische Herausforderungen tele- medizinischer Anwendungen liegen, und helfen, hierfür Lösungsansätze zu entwickeln.

\section{Studiendesign und Unter- suchungsmethoden}

Das Modellprojekt Tele-Epileptologie Ruhr (TE Ruhr) ist eine multizentrische, prospektive Pilotstudie. Primärer Endpunkt des Projektes ist die erfolgreiche technische Etablierung einer teleepileptologischen Plattform zwischen der Ruhr-Epileptologie und zunächst ausgewählten regionalen und internationalen Partnerkliniken. Für die Studie liegt ein positives Votum der Ethikkommission der Medizinischen Fakultät der Ruhr Universität Bochum vor (Rg.-Nr. 173617).

\section{Technischer Aufbau TE Ruhr}

Die TE Ruhr ist eine rein webbasierte Anwendung. Die fallbezogene Kommunikation und der Austausch von Untersuchungsdaten laufen über ein von der Firma Panvision, Essen, programmiertes Webtool, das auf einem gesicherten Server der Knappschaftskliniken Service $\mathrm{GmbH}$ gehostet wird. Das Webtool ist eine einrichtungsübergreifende elektronische Patientenakte („electronic case report form" [eCRF]), über die der konsileinholende Arzt klinische Informationen übermittelt und den Konsilauftrag stellt. Über ein integriertes Upload-Portal werden zusätzlich Untersuchungsdaten für den Konsiliarius zur Verfügung ge- 


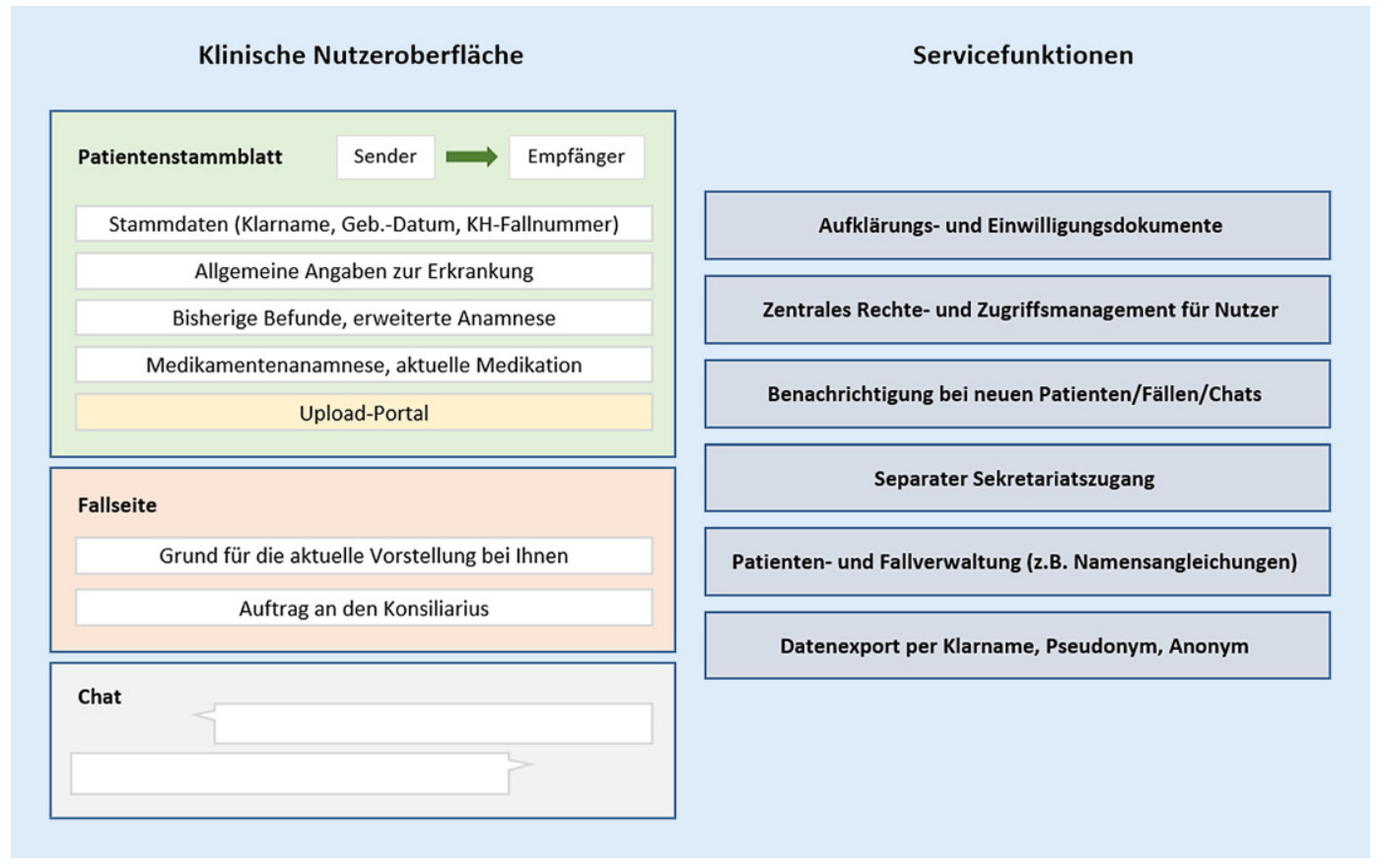

Abb. $1<$ Schematischer Aufbau der TE Ruhr stellt (s. • Abb. 1). Für Details zum technischen Aufbau der TE Ruhr s. [8] und www.tele-epileptologie.ruhr.

\section{Einschlusskriterien Partnerkliniken und Patienten}

Für den regionalen Arm der Studie wurden neurologische Abteilungen aus der Metropolregion Ruhr ausgewählt, die bereits im Vorfeld Patienten mit epileptologischen Fragestellungen in der RuhrEpileptologie vorstellten, gleiches gilt für den internationalen Arm (prächirurgische Konsultationen oder Epilepsiechirurgie, internationale Austauschprogramme).

Die ersten Kliniken wurden im September 2018 angeschlossen. Die beteiligten Abteilungen und Ärzte mussten ihr Einverständnis zur Teilnahme erklären. Der Einschluss von Patienten erfolgt nach Maßgabe der Ärzte vor Ort und schriftlicher Aufklärung der Patienten.

\section{Erhebungsinstrument der Zwischenevaluation}

Für die Zwischenevaluation wurden in den regionalen Kliniken jeweils der leitende Arzt und der projektverantwortliche Fach- oder Oberarzt gebeten, einen Fragebogen im Rahmen eines Telefonin- terviews auszufüllen, in den internationalen Kliniken nur der direkt beteiligte Arzt. Der Fragebogen wurde vor dem Telefoninterview zugestellt. Alle Interviews wurden von der Projekt-koordinierenden Autorin (S.M.) durchgeführt.

Der Fragebogen umfasste Angaben zum Krankenhaus und zur Funktion der Befragten in der Klinik.

Zur Charakterisierung der Abteilungsgröße und Anzahl der Patienten mit Epilepsien oder anderen anfallsartigen Erkrankungen wurde die Anzahl der Patienten mit den Diagnosen G40.x, G41.x, R55 oder F44.5 als Aufnahme-, Haupt- oder Nebendiagnose im 1-JahresZeitraum 01.10.19 bis 30.09.20 erfasst. Erhoben wurden die grundsätzliche Erwartung an die TE Ruhr, technische Umsetzung des Informationsaustausches, Bewertung der aktuell vorliegenden technischen Umsetzung und der erfolgten fachlichen Beratung sowie das Nutzungsverhalten. Der Fragebogen bestand aus halboffenen und offenen Fragen. Die statistische Auswertung der Interviews erfolgte deskriptiv.

\section{Ergebnisse}

Zum Zeitpunkt der Befragung waren 8 regionale und 4 internationale Kliniken
Projektpartner, in • Tab. 1 sind diese namentlich aufgeführt.

Von maximal 20 möglichen Interviews ( 2 pro regionaler Klinik $=16$, 1 pro internationaler Klinik=4) konnten 18 Interviews geführt werden. Es fehlen 2 Interviews mit leitenden Ärzten.

Die Kliniken des regionalen Armes lassen sich wie folgt charakterisieren:

- neurologische Abteilungen nicht universitärer Krankenhäuser, einmal kombiniert mit Geriatrie, einmal als Teil einer internistischen Abteilung, - neurologische Bettenzahl im Mittel 59 (19 bis 120, Median 53),

- Anzahl Patienten mit G40.x, G41.x, R55 oder F44.5 als Aufnahme-, Haupt- oder Nebendiagnose im 1-Jahres-Zeitraum (01.10.19 bis 30.09.20) im Mittel 621 Patienten (219 bis 1362, Median 555),

- Anzahl spezialisierter Epileptologen (DGfE-zertifiziert) im Team: 0.

Für die Kliniken des internationalen Armes gilt:

- 3 neurologische Abteilungen (Universitätskliniken) und 1 pädiatrische Abteilung (nicht universitäres Krankenhaus der Maximalversorgung) mit epileptologischer Expertise, 
Z. Epileptol. 2021·34:311-317 https://doi.org/10.1007/s10309-021-00425-0

(c) Der/die Autor(en) 2021

S. Mues · W. Grönheit · T. Wehner · J. Wellmer

Tele-Epileptologie Ruhr: Zwischenevaluation eines telemedizinischen Modellprojektes

\section{Zusammenfassung}

Hintergrund. Diagnose und Behandlung von Patienten mit Epilepsie oder anderen anfallsartig auftretenden Symptomen erfordert in manchen Situationen Spezialistenwissen. Um dieses auch Abteilungen ohne eigene spezialisierte Epileptologen zur Verfügung stellen zu können, wurde im Rahmen des Modellprojektes Tele-Epileptologie Ruhr (TE Ruhr) eine telemedizinische Infrastruktur zum patientenbezogenen Austausch zwischen allgemeinneurologischen Akutkliniken bzw. ausländischen Epilepsiezentren und der RuhrEpileptologie, Bochum, geschaffen.

Methoden. Die TE Ruhr ist eine multizentrische, prospektive Pilotstudie. Die telemedizinische Kommunikation inklusive Austausch von Untersuchungsdaten läuft über ein Webtool (rein webbasierte Anwendung). Es wurden Kliniken angeschlossen, mit denen bereits im Vorfeld eine Kooperation bestand. Patienten wurden nach Maßgabe der behandelnden Ärzte nach schriftlicher Einwilligung eingeschlossen. Die Zwischenevaluation wurde mit verantwortlichen Ärzten in den Kliniken per Fragebogen im Rahmen eines Telefoninterviews erstellt.

Ergebnisse. Zum Zeitpunkt der Befragung waren 8 regionale und 4 internationale Partnerkliniken angeschlossen. Die befragten Ärzte bewerten das Gesamtprojekt inklusive der technischen Realisierung mit einer Durchschnittsnote von 2,1 (1-4 auf einer Schulnotenskala von 1-6). Alle Befragten sehen einen Nutzen der TE Ruhr eher für
Einzelfälle als für Routinevorstellungen. Wichtig für die Teilnehmer ist eine einfache und schnelle Bedienung der Konsilplattform. Diskussion. Die Zwischenanalyse des Modellprojekts TE Ruhr hat gezeigt, dass die technische Etablierung einer teleepileptologischen Konsilplattform erfolgreich möglich ist. Die Ergebnisse machen über das konkrete Projekt hinaus deutlich, wo technische und organisatorische Herausforderungen telemedizinischer Anwendungen liegen und wie diesen begegnet werden kann.

Schlüsselwörter

Telemedizin · Neurologie · Smartphonevideo . MRT · EEG

\section{Tele-epileptology Ruhr: interim evaluation of a telemedical model project}

\section{Abstract}

Background. The diagnosis and treatment of patients with epilepsy or other paroxysmal neurological symptoms require specialist input in some situations. In order to make this available to departments without specialized epileptologists, a telemedical infrastructure was created for patient-related exchange between general neurological departments or foreign epilepsy centers and the Ruhr Epileptology, Bochum, Germany within the model project Tele-epileptology Ruhr (TE Ruhr).

Methods. The TE Ruhr is a multicenter, prospective pilot study. The telemedical communication including exchange of original technical data (e.g. MRI, EEG) runs via a web tool (purely web-based application). Departments with a pre-existing cooperation were invited to participate. Patients were included after written consent was obtained from the treating physicians. The interim evaluation was conducted with responsible physicians in the participating departments by questionnaire during a telephone interview. Results. At the time of the survey eight regional and four international departments were affiliated. The interviewed physicians rated the overall project, including the technical implementation, with an average grade of 2.1 (range 1-4 on a scale from 1 very good to 6 very poor). All respondents saw a benefit of TE Ruhr for individual cases rather than for routine presentations. Important for the participants was a simple and fast handling of the consultation platform.

Discussion. The interim analysis of the model project TE Ruhr has shown that the technical establishment of a tele-epileptological consultation platform is successfully possible. The results illustrate the technical and organizational challenges of telemedical applications and how these can be met, beyond this particular project.

\section{Keywords}

Telemedicine - Neurology · Smartphone video . $\mathrm{MRI} \cdot \mathrm{EEG}$
- bezüglich Bettenanzahl und Anzahl Patienten mit oben genannter Diagnose liegen keine vergleichbaren Angaben vor.

Die befragten Ärzte bewerten das Gesamtprojekt mit einer Durchschnittsnote von 2,1 (Noten 1 bis 4 genannt) auf einer Notenskala von 1 (sehr gut) bis 6 (ungenügend).

Im Zeitraum 01.09.2018 bis 30.09.2020 wurden 105 Konsile bearbeitet. Die regionalen Kliniken haben 0 bis 19 (Mittelwert 6) Konsile, die internationalen
Partnerkliniken von 2 bis 58 (Mittelwert 17) Konsile gestellt. Nachfolgend werden die Ergebnisse der einzelnen Aspekte der Zwischenevaluation dargestellt.

\section{Grundsätzliche Erwartung an die TE Ruhr und aktuelle Bewertung}

Die vom telemedizinischen Anschluss an die Ruhr-Epileptologie erhofften Vorteile unterscheiden sich zwischen regionalem und internationalem Arm. Regionale Partnerkliniken erwarteten eine enge- re und unkomplizierte Anbindung an „ihr“ Referenzzentrum. Durch fallbezogene Empfehlungen erhofften sie sich eine bessere Patientenversorgung und einen Wissenszuwachs. Von 14 Ärzten sahen 8 die in die TE Ruhr gesetzten Erwartungen bestätigt, 3 teilweise bestätigt, ein/e Arzt/Ärztin nicht. Letztere/r empfindet das Webtool zu kompliziert und zeitaufwendig. Zwei Ärzte hatten zum Zeitpunkt des Interviews noch keine Patienten vorgestellt. Die internationalen Partnerkliniken erwarteten einen spezifischen Austausch über bestimmte 
Tab. 1 Partnerkliniken Tele-Epileptologie

Ruhr

Regionaler Arm (neurologische Abteilun-

gen)

St. Josef-Krankenhaus Essen-Kupferdreh

St. Marien-Hospital Lünen

Sana Klinikum Remscheid

Klinikum Vest Recklinghausen

Elisabeth Krankenhaus in Recklinghausen

Evangelisches Krankenhaus Castrop-Rauxel (seit 03/2020)

Klinikum Dortmund (seit 06/2020)

Paracelsus-Klinik Hemer (seit 05/2020)

Internationaler Arm

Universitäres Epilepsiezentrum Tartu (Estland)

Universitäres Epilepsiezentrum Vilnius (Litauen)

Universitäres Epilepsiezentrum Bangkok (Thailand)

Pädiatrische Abteilung mit epileptologischer Expertise, Hospital "Sf.Ioan cel Nou“, Suceava (Rumänien)

epileptologische Fragestellungen. Hierdurch sollte die Expertise in der eigenen Klinik erhöht werden. Zwei von 4 Ärzten gaben an, dass sich die Hoffnungen erfüllt haben, 2 sahen ihre Hoffnungen teilweise erfüllt.

Als generelle Befürchtungen im Vorfeld des Projektes wurden von 3 Ärzten des regionalen Armes technische Schwierigkeiten benannt, wobei diese nicht näher spezifiziert wurden. Sie gaben an, dass sie zumindest am Anfang des Projektes technische Probleme (Übertragung der EEG-Untersuchungen) hatten. Ein Arzt/Ärztin befürchtete, dass sich die Fragestellungen im klinischen Alltag nicht ergeben und sieht dies im Projektverlauf bestätigt. Die einmal geäußerte Befürchtung, sich mit etwas Neuem/Unbekannten auseinandersetzen zu müssen, wird im Projektverlauf relativiert („wenn man sich damit auseinandersetzt, klappt es schon“). Von den Partnern des internationalen Armes werden als generelle Befürchtungen je 1-mal lokale technische Probleme mit dem Upload der Originaldaten und datenschutzrechtliche Aspekte genannt. Beide Befürchtungen haben sich im Projektverlauf bestätigt, folgende Lösungen wurden gefunden:
- Nutzung paralleler Videokonferenzen, in welchen Originaldaten per geteiltem Bildschirm ausgetauscht werden,

- anonymisierte Vorstellung der Patienten, weil Übersetzung der Einwilligungsunterlagen in Originalsprache noch ausstehend.

Die - Abb. 2 gibt eine Übersicht über die erwarteten Konsilgründe, die sich zwischen regionalen und internationalen Partnerkliniken unterscheiden.

\section{Technische Umsetzung des Informationsaustausches}

Fragen hierzu wurden nur den 8 regionalen und 4 internationalen Ärzten gestellt, die das Tool auch praktisch nutzten.

\section{Einrichtungsübergreifende elektronische Patienten- und Fallakte (eCRF)}

Die Mehrheit aller Ärzten findet eine Kombination von Auswahlfragen und Freitextformulierungen zur Übermittlung klinischer Informationen und der Fragestellung sinnvoll (s. - Abb. 3a). Für die Medikamentenanamnese gibt es keine eindeutige Präferenz. Mit den Ausfülloptionen des eCRF und dem hierfür verbundenen zeitlichen Aufwand sind die befragten Ärzte überwiegend zufrieden (s. $\bullet$ Abb. 3c, d).

\section{Austausch von Untersuchungs- daten}

Alle Ärzte erwarten, dass ein teleepileptologisches Tool die Übertragung von EEG und bildgebenden Untersuchungen ermöglicht. Für alle Ärzte des internationalen Armes ist die Übertragung von Videos und Dokumenten relevant, im regionalen Arm für 4/8 (Videos) bzw. 5/8 (Dokumente). Bezüglich der Umsetzung der TE Ruhr mit implementiertem Upload-Portal sehen 8/12 Befragten ihre Anforderungen im klinischen Alltag erfüllt. Die $\bullet$ Abb. 3b zeigt die Einschätzung der Funktionalität und das Nutzungsverhalten für einzelne Datenformate.

Allerdings übertrug keine der regionalen Kliniken im Auswertungszeitraum Bildgebungsdaten über das Webtool, stattdessen wurden die eta- blierten Leitungen des Westdeutschen Teleradiologie Verbunds (TRV) genutzt. Der große Anteil des regelhaft nicht durchgeführten Uploads von Videos ist ebenfalls auf die regionalen Partnerkliniken zurückzuführen.

\section{Persönlicher Austausch}

Zehn von 12 Ärzten finden die Möglichkeit zum persönlichen Austausch über die Chatfunktion des Webtools sinnvoll. Darüber hinaus wünschen sich aber 7/12 Ärzten Telefonkontakte bzw. 2/12, beide internationaler Arm, Videotelefonate.

\section{Inhaltliche medizinische Beratung}

Hinsichtlich der inhaltlichen Beratung gaben die befragten Ärzte an, über die TE Ruhr Fragen zu stellen, die sich im stationären (18/18) oder im ambulanten (1/18) klinischen Alltag ergeben. Die Mehrheit der Ärzte gab an,

- dass die Konsilanfragen hinreichend schnell beantwortet wurden (13/18) und

- dass sie mit der Qualität der Antworten einverstanden seien (14/18).

\section{Analyse des Nutzungsverhaltens}

Die relative seltene Nutzung der TE Ruhr läge daran, dass

- sich der Einschätzung der befragten Ärzte nach komplizierte Fragestellungen im Bereich Epileptologie/ Epilepsiechirurgie im klinischen Alltag nicht häufig ergeben würden $(10 / 18)$,

- der technische Ablauf zu kompliziert sei $(5 / 18)$,

- die Konsilanforderung inklusive Übertragung der Zusatzdiagnostik zu zeitintensiv sei (5/18).

Als weitere Argumente für eine Nichtoder seltene Nutzung wurden fehlende Routine mit dem Webtool, Probleme mit der eigenen IT-Abteilung, ausreichende eigene Expertise, Angst vor Offenbarung von Wissenslücken und datenschutzrechtliche Probleme benannt. Bei einer Klinik aus dem internationalen Arm gab es Verzögerungen in der Beantwor- 


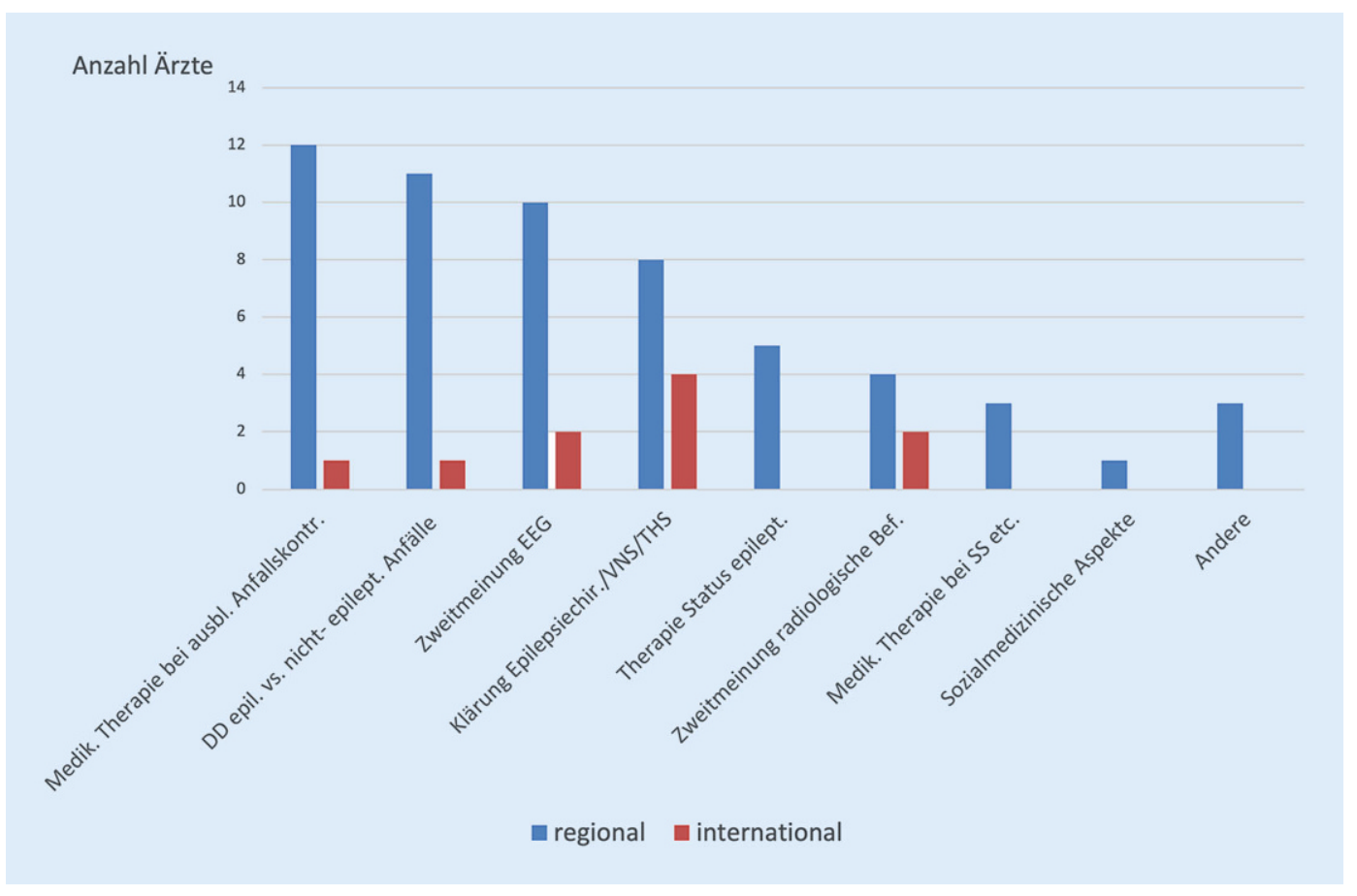

Abb. $2 \varangle$ Konsilgründe, bezogen auf 16 Ärzte in regionalen und 4 Ärzte in internationalen Partnerkliniken. Mehrfachnennungen waren möglich

tung erster Fälle, weshalb im Verlauf die Motivation abgenommen habe.

Alle Befragten sehen für Einzelfälle weiterhin einen Nutzen der TE Ruhr. Als Verbesserungsvorschläge wurden genannt:

- die Vereinfachung und zeitliche Optimierung der Konsilanfrage,

- Schaffung einer finanziellen Winwin-Situation (finanzielle Vergütung für konsileinholenden Arzt und Konsiliarius).

\section{Diskussion}

Die Zwischenauswertung des Pilotprojekts TE Ruhr zeigt, dass die Etablierung einer interhospitalen Konsilplattform für umfassende epileptologische Fragestellungen technisch erreichbar ist. Im Gegensatz zu den telemedizinischen Lösungen zur Versorgung von Schlaganfallpatienten, bei denen neben Bilddaten nur überschaubare Mengen klinischer Informationen übermittelt werden, kann die TE Ruhr komplexe klinische Informationen mittels eCRF, technische Untersuchungen wie EEG und Video-EEG, Anfallsvideos und weitere Datenformate per Upload-Portal und patientenbezogene Kommunikation über eine ChatFunktion übertragen. Die gewählte webbasierte Anwendung [8] hat den Vorteil der einfachen Installierbarkeit und der ortsunabhängigen Nutzbarkeit auf jedem Endgerät.

Die Befragung der Teilnehmer liefert Hinweise darauf, worin sie im klinischen Alltag einen Gewinn sehen und wo Erwartungen bislang nicht erfüllt wurden. Hierbei sind die Unterschiede zwischen regionalen und internationalen Partnerkliniken zu berücksichtigen: In den regionalen Partnerkliniken arbeiten keine spezialisierten Epileptologen im Team. Hiermit und auch anhand der weiteren erhobenen Charakteristika entsprechen diese den durchschnittlichen neurologischen Abteilungen nichtuniversitärer Häuser [6], somit können die Ergebnisse unserer Studie vermutlich über die beteiligten Kliniken hinaus verallgemeinert werden.

Bei den internationalen Projektpartnern handelt es sich um spezialisierte Epileptologen, die sich bezüglich epilepsiechirurgischer Behandlungsoptionen austauschen wollen und Hilfe bei der Kompensation technischer Limitierungen (z.B. EEG-Quellenlokalisation, MRT-Postprocessing) wünschen. Ihre Gruppe ist klein, daher sind ihre Einschätzungen weniger repräsentativ.

Über beide Nutzergruppen hinweg fällt die Gesamtbewertung des Projektes positiv aus. Insbesondere für das Setting regionaler Partnerkliniken ist jedoch für unser Projekt und vergleichbare Anwendungen festzuhalten, dass

- der zeitliche und technische Aufwand für die Konsilstellung dem klinischen Alltag angepasst sein muss,

- Indikationen zur Vorstellung von Patienten über das telemedizinische Instrument seltener gesehen werden, als aus der Sicht eines Epilepsiezentrums erwartet.

Für beide Gruppen gilt, dass

- eine gewisse Variabilität bei den verwendeten Kommunikationswegen für die Nutzer wichtig ist.

Der zeitliche Aufwand für die Nutzung einer komplexen telemedizinischen Plattform ist nur begrenzt minimierbar. Die Teilnehmer wurden bei der Einarbeitung darauf hingewiesen, dass nur für die Fragestellung relevante eCRF-Felder auszufüllen sind. Dennoch wurde von knapp $30 \%$ der Teilnehmer der benötigte Zeitaufwand für zu hoch gehalten. Als Gründe kommen auch mehrfach abgefragte Vidierungen sowie das Einholen umfassender Patienteneinverständniserklärungen in Betracht. Beides ist jedoch erforderlich, um die Vorgaben der Datenschutzgrundverordnung (DSGVO) zu erfüllen. Diese Formalitäten laufen 


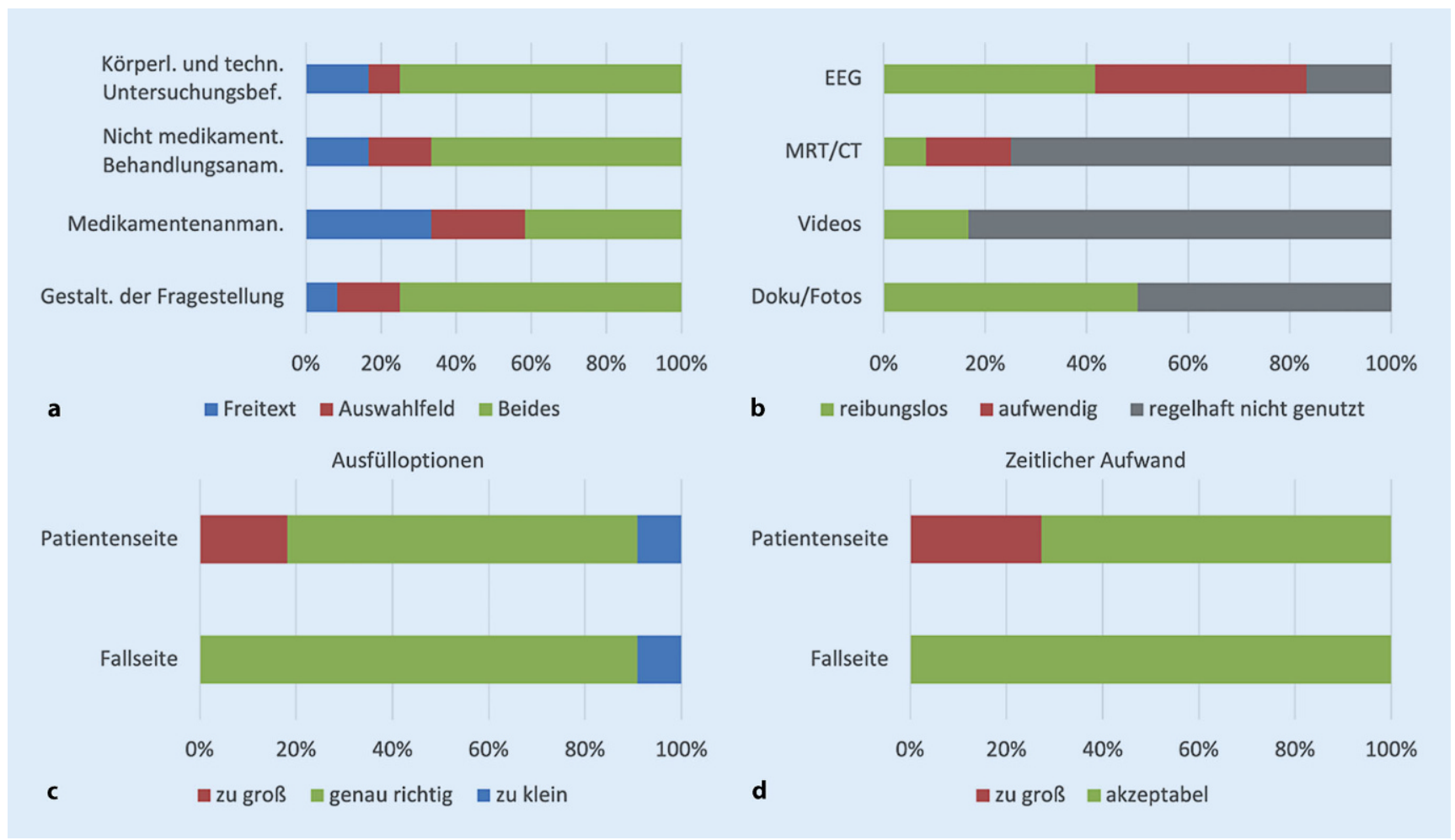

Abb. 3 A Technische Präferenzen und Erfahrungen mit der TE Ruhr - gepoolte Angaben regionaler und internationaler Arm. a Bevorzugte Eingabemöglichkeit für Krankengeschichte und Fragestellung im eCRF. b Funktionalität bzw. Nutzungsverhalten des implementierten Datenuploads, unterteilt nach Datenformaten. c, d Allgemeine Zufriedenheit mit dem Umfang der Ausfüllmöglichkeiten und dem zeitlichen Aufwand, getrennt nach Patientenstammblatt-und Fallseite. Angaben entsprechen dem Anteil der befragten Ärzte in Prozent

dem klinischen Bedürfnis nach einer einfachen und schnellen Verwendung des Instruments entgegen, um „nur mal gerade“ eine Zweitmeinung zu einem Fall einzuholen. Das Problem ist nicht TE Ruhr-spezifisch. Die deutlich schnellere Alternative des Telefonanrufes bei persönlich bekannten Spezialisten entspricht aber nicht in allen Fällen den Anforderungen der DSGVO [2] und erst recht nicht der ärztlichen Dokumentationspflicht. Hier wäre projektübergreifend wichtig, letztlich auch im Patienteninteresse, praktikable Minimalstandards für Patientenaufklärungen bei telemedizinischen Lösungen zu definieren.

Wo immer technische oder LayoutVereinfachungen die Nutzung beschleunigen können, sollte diese Möglichkeiten genutzt werden. Wir haben die Rückmeldungen der Teilnehmer für Änderung des eCRF-Designs und der Organisation des Fallblattes (jetzt: „aktueller Konsilauftrag“) genutzt. Der Effekt dieser Maß- nahme wird erst im Rahmen der finalen Evaluation erfasst werden können.

Die Möglichkeit der Verwendung alternativer Datenrouten kann unter Umständen ebenfalls zur Beschleunigung des Konsilprozesses beitragen. Die (delegierte) Versendung von Bildgebungsdaten via TRV statt über das Upload-Portal führt zu keinerlei Dokumentationsverlust oder Sicherheitsbedenken. Problematisch ist das bei der Videotelefonie, weshalb dieser parallele Weg nicht empfohlen wird.

Selbst bei optimaler technischer Organisation bleibt dennoch die Frage der Nutzungsfrequenz. Allgemeine Nutzungsvorbehalte gegenüber dem Instrument, das Fehlen von Nutzungsroutine und noch nicht gemachte positive Erfahrungen können überwunden werden nicht zuletzt durch eine persönliche Betreuung der Partnerkliniken und regelmäßige Anwendertreffen.

Problematischer kann sein, dass der Nutzen einer telemedizinischen Anfrage nur in komplexen Einzelfällen gesehen wird. Dabeilegen verschiedene Daten nahe, dass auch bei der vermeintlich einfachen Fragestellung wie „erster Anfall“ Expertenwissen helfen kann. Sogenannte "first seizure clinics“ helfen, den Anteil von Fehldiagnosen zu verringern $[3,5]$. Telemedizinische Anwendungen wie die TE Ruhr wären prädestiniert, diese ohne großen Aufwand flächendeckend anzubieten.

Es bedarf jedoch der Entwicklung genauerer Standards für regionale Netzwerke, welche Patienten vorgestellt werden und welche Ärzte teilnehmen sollten. Und es muss die Refinanzierung ärztlicher Leistungen sichergestellt werden sowohl auf der Seite des/der konsileinholenden als auch auf der Seite des/der konsilbeantwortenden Arztes/Ärztin, damit beide den erforderlichen Personalaufwand refinanzieren können.

Ein wesentlicher Teil der Erfolgsgeschichte der Schlaganfall-Telemedizin ist sicherlich darauf zurückzuführen, dass ihre Einführung zu einer Vorgabe von Fachgruppen und Zertifizierungsgremi- 
en [4] gemacht wurde, da ihr Nutzen in verschiedenen Teilaspekten belegt werden konnte [1, 7]. Möglicherweise können auch in der Epileptologie Vorgaben von Fachgesellschaften die Nutzung telemedizinischer Instrumente fördern, wenn ein Nutzen für die Patienten belegt werden kann.

\section{Fazit für die Praxis}

- Die Zwischenanalyse der TE Ruhr zeigt, dass die regionale und internationale Etablierung einer teleepileptologischen Konsilplattform technisch und DSGVO-konform möglich ist.

- Ein zeit- und ärztliche Ressourcen sparender Ablauf des teleepileptologischen Konsils sind nach den Angaben der Nutzer wichtig für eine Akzeptanz und letztlich auch Anwendung im Alltag.

- Über epileptologische Fortbildungen sollten nichtspezialisierte Kliniken für Fälle sensibilisiert werden, in denen eine Einbindung von Spezialisten sinnvoll ist.

\section{Korrespondenzadresse}

\section{Dr. med. Sigrid Mues}

Ruhr-Epileptologie, Klinik für Neurologie, Universitätsklinikum Knappschaftskrankenhaus Bochum, Ruhr-Universität Bochum In der Schornau 23-25, 44892 Bochum, Deutschland

sigrid.mues@kk-bochum.de

Danksagung. Wir danken der Werner Richard-Dr. Carl Stiftung für die finanzielle Förderung des Projekts und bei den Projektpartnern der Firma VISUS (Dr. M. Kämmerer), der Firma MedEcon Telemedizin $\mathrm{GmbH}$ (M. Kremers), der Firma BESA (A. Ebert und T. Scherg) und der Knappschaftskliniken Service $\mathrm{GmbH}$.

Funding. Open Access funding enabled and organized by Projekt DEAL.

\section{Einhaltung ethischer Richtlinien}

Interessenkonflikt. S. Mues, W. Grönheit, T. Wehner und J. Wellmer geben an, dass bezüglich der in der Danksagung genannten Institutionen und Firmen keine Interessenkonflikte bestehen, die über die Studienunterstützung bzw. Kooperation hinausgehen.
Für die aufgeführten Studien gelten die jeweils dort angegebenen ethischen Richtlinien.

Open Access. Dieser Artikel wird unter der Creative Commons Namensnennung 4.0 International Lizenz veröffentlicht, welche die Nutzung, Vervielfältigung, Bearbeitung, Verbreitung und Wiedergabe in jeglichem Medium und Format erlaubt, sofern Sie den/die ursprünglichen Autor(en) und die Quelle ordnungsgemäß nennen, einen Link zur Creative Commons Lizenz beifügen und angeben, ob Änderungen vorgenommen wurden.

Die in diesem Artikel enthaltenen Bilder und sonstiges Drittmaterial unterliegen ebenfalls der genannten Creative Commons Lizenz, sofern sich aus der Abbildungslegende nichts anderes ergibt. Sofern das betreffende Material nicht unter der genannten Creative Commons Lizenz steht und die betreffende Handlung nicht nach gesetzlichen Vorschriften erlaubt ist, ist für die oben aufgeführten Weiterverwendungen des Materials die Einwilligung des jeweiligen Rechteinhabers einzuholen.

Weitere Details zur Lizenz entnehmen Sie bitte der Lizenzinformation auf http://creativecommons.org/ licenses/by/4.0/deed.de.

\section{Literatur}

1. Audebert HJ, Kukla C, Vatankhah B, Gotzler B, Schenkel J, Hofer S, Fürst A, Haberl RL (2006) Comparison of tissue plasminogen activator administration management between Telestroke Network hospitals and academic stroke centers: the Telemedical Pilot Project for Integrative Stroke Care in Bavaria/Germany. Stroke 37:1822-1827

2. Eickmeier $F$ (2018) Der rechtliche Rahmen für Datenschutz bei E-Health. In: Bauer C, Eickmeier F Eckard M (Hrsg) E-Health: Datenschutz und Datensicherheit. Springer Gabler, Wiesbaden, S 45-52

3. Hamiwka LD, Singh N, Niosi J, Wirrell EC (2007) Diagnostic inaccuracy in children referred with "first seizure": role for a first seizure clinic. Epilepsia 48:1062-1066

4. Hubert GJ, Santo G, Vanhooren G, Zvan B, Tur Campos S, Alasheev A, Abilleira S, Corea F (2019) Recommendations on telestroke in Europe. Eur Stroke J4:101-109

5. Jackson A, Teo L, Seneviratne U (2016) Challenges in the first seizure clinic for adult patients with epilepsy. Epileptic Disord 18:305-314

6. Ministerium für Arbeit, Gesundheit und Soziales des Landes Nordrhein-Westfalen https://www. mags.nrw/krankenhausdatenbank. Zugegriffen: 20.Apr. 2021

7. Moustafa H, Barlinn K, Prakapenia A, Winzer S, Gerber J, Pallesen LP, Siepmann T, Haedrich K, Wojciechowski C, Reichmann H, Linn J, Puetz V, Barlinn J (2021) Endovascular therapy for anterior circulation large vessel occlusion in telestroke. J Telemed Telecare 27:159-165

8. Mues S, Hamer HM, von Podewils F, Sotoodeh A, Rosenow F, Wellmer J, Zöllner JP (2021) Telemedizin in der Epilepsieversorgung: Arzt-zuArzt-Anwendungen, Teil II: Aktuelle Projekte in Deutschland.ZEpileptol. https://doi.org/10.1007/ s10309-021-00426-z
In eigener Sache

Autoren

WERKSTATT

GRATIS

Ein Service für Autoren von Springer Medizin

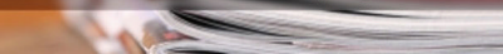

Fortbildungen für

Autor*innen und Gutachter*innen

Die ersten Veröffentlichungen sind für Jeden ein wichtiger Schritt in der angestrebten Karriere. Wissenschaftliche Artikel sind entscheidend dafür, dass die eigene Arbeit in der Community wahrgenommen wird. Es geht darum, die eigenen Ideen national und international auszutauschen und sicherzustellen, dass die Ergebnisse Wirkung erzielen.

Die Online-Kurse der Autorenwerkstatt helfen, sich leicht einen Überblick über das Schreiben, Einreichen, Begutachten und Veröffentlichen eines Manuskripts zu verschaffen.

5 Online-Kurse zu den wichtigsten Standards des wissenschaftlichen Publizierens:

- Wie verfasse ich ein Manuskript?

- Writing in English für deutschsprachige Autorinnen und Autoren

- Wie funktionieren Publikation und Begutachtung?

- Anleitung zur Open-AccessVeröffentlichung

- Leitfaden zur Peer-ReviewBegutachtung

Für alle, die auf SpringerMedizin.de registriert sind!

Jetzt gratis fortbilden unter www.springermedizin.de/ autorenwerkstatt/ 